\title{
The Theory of Essence. III: Neuroanatomical and Neurophysiological Aspects of Interactionism
}

\author{
J. Kenneth Arnette, Ph.D. \\ Eastern Washington University
}

\begin{abstract}
This article continues the construction of a dualistic interactionist theory of the near-death experience (NDE), the theory of essence, which was begun in two previous articles (Arnette, 1992, 1995). The present work represents an extension of the theory to the microscopic level of analysis, in order to specify in detail the mechanism of essence-brain interaction and to address some general and specific objections to interactionism and the theory of essence. In the theory construction process, a second issue is addressed: that of the apparent multiplicity of causes of NDEs or NDE-like experiences. I show that this multiplicity is simply a manifestation of the mode of essence-brain interaction and is accurately predicted by the theory.
\end{abstract}

How can thoughts and ideas, decidedly abstract entities, arise from mere neurons or collections thereof? Conversely, how do thoughts influence neurons and, thereby, the functioning and actions of our bodies? These questions are a modern rendering of an ancient dilemma, the philosophical mind/body problem, which inquires as to the nature of our conscious experience and indeed of our very humanity. The present work, the third in a series of related articles, approaches these questions from the context of information provided by the near-death experience (NDE) and, in the process, sheds theoretical light on the apparent multiplicity of causes of NDEs and NDE-like phenomena.

In two previous articles (Arnette, 1992, 1995a) I put forth components of an explanatory theory of the NDE, the theory of essence, built

J. Kenneth Arnette, Ph.D., is Assistant Professor of Psychology at Eastern Washington University. This work was initiated while Dr. Arnette was a graduate student in the Department of Psychology, Colorado State University, Fort Collins, CO. Reprint requests should be addressed to Dr. Arnette at the Department of Psychology, MS-94, Eastern Washington University, 526 Fifth Street, Cheney, WA 99004-2431. 
upon an empirical foundation provided by the NDE data. This theory is a form of mind/body dualism called dualistic interactionism, holding that humans are composed of two parts comprising two different substances-the physical body composed of matter, and the essence composed of a nonmaterial substance-which interact during physical life to produce mind.

In the first article (Arnette, 1992), hereafter referred to as Part I, I explored the similarities between the predictions of Albert Einstein's (1961) theory of general relativity and certain aspects of the NDE (Moody, 1975), such as the tunnel, timelessness, and weightlessness. I interpreted the NDE from a relativistic perspective that yielded, for example, the notion that the NDE tunnel is identical to the wormhole (Misner, Thorne and Wheeler, 1973), providing a pathway to other universes.

In the second article (Arnette, 1995a), hereafter referred to as Part II, I proposed a connection between essence/body interactions as described by NDErs and the interactions between electric dipoles as described in electromagnetism and quantum mechanics. For example, I proposed that the NDE phenomenon of thought reception (Moody, 1975) was analogous to the radiationless process of fluorescence energy transfer (Stryer, 1978). In this manner, I interpreted additional elements of the NDE within the framework of physics and chemistry, providing a level of organization and explanation of the near-death phenomena that materialistic theories have thus far been unable to offer.

In the present article I continue the construction of the theory of essence and present an extension of the theory to the microscopic level of analysis, in order to specify in more detail the mechanism of essence/ brain interaction and to address some general objections to interactionism, as well as some specific objections to the theory of essence. As I will discuss below, the lack of an interaction mechanism has long been an objection posed by materialists to interactionism. In the process of constructing this mechanism, I will address a second issue: the apparent multiplicity of causes of NDEs or NDE-like experiences. I will show that this multiplicity is simply a manifestation of the mode of essence/brain interaction.

\section{The Perceived Inadequacies of Interactionism}

In the centuries-old argument between materialists and dualistic interactionists over the mind/body problem, materialists have identified the violation of scientific conservation principles and the lack of a 
$\mathrm{mind} / \mathrm{brain}$ interaction mechanism as the key weaknesses in the interactionist position (Broad, 1925; Cornman, 1981; Taylor, 1992). Hermann von Helmholtz was the first to propose that a dualistic conception of mind is ruled out by the principle of the conservation of energy (Hergenhahn, 1986), which states that "[e]nergy may be transformed from one kind to another, but it cannot be created or destroyed; the total energy is constant" (Halliday and Resnick, 1970, p. 126). For systems such as living organisms, the relevant principle is the first law of thermodynamics, which incorporates the energy conservation principle and states in part that a change in the energy of the system is accompanied by a change, of equal magnitude and opposite sign, in the energy of the surroundings, so that the total energy of the system plus surroundings (that is, a closed system) remains constant (is conserved) for any process (Levine, 1978).

James Cornman (1981) stated the apparent incompatibility of dualistic interactionism and energy conservation as follows: supposed mental states, such as a pain or volition, in interacting with the brain, should result in an increase in the total energy of the affected neurons through their firing; there can be no corresponding mental energy loss, however, because the presumed nonphysical mind has no mass, and therefore no energy. Thus, if interactionism were true, a net increase of energy in the brain would occur and the energy conservation principle would be violated for the closed system consisting of the universe.

Many years earlier, C.D. Broad (1925) had answered this objection, suggesting that the mind acts on the synapses of the brain by changing their resistances and thus directing the flow of neural currents without adding or subtracting energy to or from the physical system. In this way, Broad proposed, the mind could act causally on the brain and thus influence behavior. Cornman (1981) recited Broad's argument and replied that Broad had ignored another conservation principle, the conservation of linear momentum, and its concomitant problems for interactionism.

Cornman's (1981) view of this issue was that by selecting one neural pathway over another, the mind would change the direction of the neural impulse, and thus the total linear momentum of the brain. But since linear momentum must be conserved in the absence of the influence of an outside, physical force (Halliday and Resnick, 1970), and since the nonphysical mind presumably has neither mass nor electrical charge, no mental phenomenon can exert such a force in the brain. Thus the conservation of linear momentum, if not energy, poses an unsolvable problem for the interactionist, according to Cornman and many other materialists. 
With respect to these historical objections to interactionism, a goal of the present work is to show that these objections are based on two classes of misconceptions. First, the interactionist position has generally been misunderstood, painting dualists into an unnecessarily small and rigid corner concerning interactionist possibilities. Second, Cornman (1981) has misapplied principles from physics due to some inaccurate notions of neurophysiology.

In addition to these general, historical objections to interactionism, in Parts I and II of this model I drew specific objections from two sources. First, V. Krishnan (1999) correctly pointed out that many questions and issues concerning the theory of essence went unanswered in the first two articles. Among Krishnan's comments were the following: (1) since the body and the essence can share some properties, the theory is not truly dualistic and the essence is actually material; (2) science does not know what matter really is, and therefore we cannot say what matter is not; (3) something that is not matter could not generate an electric field; (4) a substance with an electric field could not be free from gravity; and (5) it is unclear how the essence comes into existence. These issues show the need for the further explication of the theory of essence presented below.

Juan Gómez-Jeria and Carlos Madrid-Aliste (1996) provided a second source of objections to the theory of essence. In their article discussing the relationship between evolution and human consciousness, these authors asserted that

[i]f we accept that consciousness is a nonphysical thing ...then we need to answer scientifically when nonphysical consciousness or nonphysical brain properties appeared... The next question to answer is why they appeared at [a given] stage and not a previous one. The only reasonable scientific answer would be because at [a given stage] the physical structures of the central nervous system made it possible (p. 260; italics are the authors').

But this line of reasoning is unavailable to the dualist, the authors claimed, because ultimately the nonphysical mind would have to possess physical properties and a causal relationship with the physical body. Thus, they concluded that

human [consciousness] states exist because of the particular form of brain evolution attained in Homo sapiens sapiens. Within this context, Kenneth Arnette's (1992) attempt to revive dualism by using NDE data is untenable. (p. 261)

The authors thus (1) perpetuated the unnecessarily restrictive definition of dualism, as I will discuss below; (2) implied that the nonphysical 
mind necessarily arose within this universe; and (3) implied that a dualistic theory is fundamentally incompatible with evolutionary theory, and perhaps also with neuroscience in general. Nothing could be further from the truth. In the present article, I will show, among other points, that the theory of essence is completely compatible with both neuroscience and the evolution of human consciousness.

\section{The NDE: A Multiplicity of Causes?}

Two recent materialistic theories of the NDE have attempted to explain the fact that the NDE, or at least experiences somewhat similar to the NDE, can be triggered by a variety of events, such as psychological trauma, the effects of drugs, or traumatic physical injury. Juan Saavedra-Aguilar and Gómez-Jeria (Saavedra-Aguilar and GómezJeria, 1989; Gómez-Jeria and Saavedra-Aguilar, 1994) have proposed a physiological model of the NDE that they claim unites the biological and psychological domains and integrates the various triggers. Their model hypothesizes that a traumatic event triggers a stress response in the brain, which causes the release of certain neurotransmitters. This, along with the accompanying decrease of oxygen availability in the brain, affects the limbic system, creating abnormal excitation in limbic tissues and eventually leading to temporal lobe dysfunction similar to that found in temporal lobe epilepsy. The features of the NDE are caused by "afterdischarges propagating through limbic connections ... towards more distant regions" (Saavedra-Aguilar and Gómez-Jeria, 1989, p. 212).

This model has not been challenged in the literature, and yet is open to serious criticism in that it completely ignores the medical research of Michael Sabom (1982) and Melvin Morse (Morse, 1983; Morse, Conner and Tyler, 1985; Morse, Castillo, Venecia, Milstein and Tyler, 1986; Morse, Venecia and Milstein, 1989; Morse and Perry, 1990). These research results demonstrated that, although the various triggers can induce NDE-like experiences, it is nonetheless true that psychological stress is not a causative factor for the NDE; that hypoxia or hypercapnia, drugs, neurotransmitters, and neuropeptides are not causative factors; and that the features of the NDE and of temporal lobe epilepsy are only partly compatible, with most of the key features of the NDE completely missing in epilepsy. Furthermore, although the authors claimed to address this issue (Gómez-Jeria and Saavedra-Aguilar, 1994), the problem of accurate visual perceptions by NDErs (Sabom, 1982; Morse 
and Perry, 1990) remained unexplained in their model. This point is crucial, since no materialistic theory to date has been able to explain these observations (Arnette, 1994; 1995a; 1995b).

Morse and his colleagues (Morse, 1983; Morse, Conner and Tyler, 1985; Morse, Castillo, Venecia, Milstein and Tyler, 1986; Morse, Venecia and Milstein, 1989; Morse and Perry, 1990) proposed a physiological/ genetic model that bore some similarity to that of Saavedra-Aguilar and Gómez-Jeria (Saavedra-Aguilar and Gómez-Jeria, 1989; Gómez-Jeria and Saavedra-Aguilar, 1994). Morse's model proposed that a variety of factors, such as psychological stress, psychoactive drugs, hypoxia or hypercapnia, or direct electrical stimulation, result in disinhibition of the temporal lobe of the cortex. This, in turn, activates a genetic program built into the structure of the temporal lobe, causing the person to perceive out-of-body experiences (OBEs) and/or NDEs, which are then recorded as memories (Morse, Venecia, and Milstein, 1989).

This model possesses three apparent strengths: (1) it integrates various suggested causes of NDE-like experiences into a single model; (2) these suggested causes are not dependent on one another in a chain of events, but rather can act independently to cause the NDE; and (3) temporal lobe dysfunction is abandoned as an explanation, and a genetic program is instead invoked. The genetic component of that model preserves the uniformity of the core NDE (Moody, 1975; Ring, 1980; Sabom, 1982).

Morse's model, however, was not as promising as it initially appeared. First, it ignored the results of Morse's own research, in which he had found that stress, drugs, and hypoxia or hypercapnia were insufficient to explain the NDE. Second, as with other materialistic models, the accuracy of NDE visual observations remained unexplained. Additionally, his model, like all materialistic models, was vulnerable to two general objections: (1) there is no physiological basis for retaining the memory of the NDE (Arnette, 1994; 1995b); and (2) since the NDE offers no survival advantage, there can be no genetic or evolutionary basis for the experience (Arnette, 1995b).

As a final note on Morse's model, even its authors acknowledged its inconclusiveness. After summarizing their model, they concluded that

it is just as likely that such an area [temporal lobe] represents the seat of the soul, an area of our brain that serves as a trigger point for the release of the soul at death. (Morse, Venecia, and Milstein, 1989, p. 51)

Thus, while describing their materialistic NDE model, Morse and colleagues admitted that a decidedly dualistic model is at least as good a fit to the data. As I will show, this "seat of the soul hypothesis" (Morse 
and Perry, 1990) is consistent with the theory of essence and is much more promising than materialistic theories.

\section{Two Fundamental Questions}

\section{A Definition of Dualism}

In Part II, I asserted that because essence is not composed of matter, it does not have the exclusionary property of matter. It is therefore possible for essence and body to occupy the same three-dimensional space. In addition, essence can have location and possess shape. Consequently, I conceive essence as occupying the same space as the body and being bound to it in such a way as to move with it and interact with it in space and time. The key notion behind this body/essence binding and interaction is electromagnetism. Just as the body generates an electromagnetic field that varies in time and extends in space, so too, I posit, does the essence. The electromagnetic properties of body and essence allow the binding of each to the other, in a manner analogous to dipole/dipole attraction (Arnette, 1995a; Jackson, 1975; Lorrain and Corson, 1970). Further, in the bound state the respective electromagnetic fields intermingle and interact, allowing reciprocal causal influence between essence and brain. This is analogous to the phenomenon of dipolar rotational relaxation (Arnette, 1981, 1995a).

But this picture of interaction has been met with a question of some importance, which may be stated as follows: does not the proposal that the essence possesses physical qualities, such as an electromagnetic field, violate the prime tenet of dualism, which is that the mental substance is nonphysical (Gómez-Jeria and Madrid-Aliste, 1996; Krishnan, 1999)? This question goes to the heart of what is meant by the term "dualism."

The problem of dualistic interaction has traditionally been stated very simply as follows (Taylor, 1992): if the brain is physical and the mind is nonphysical, then how can the brain and mind interact? This is intended to be a rhetorical, unanswerable question, refuting interactionism. But if one examines this argument, it becomes evident that the premises contain the conclusion. That is, this usage of the term "nonphysical" implicitly defines a nonphysical entity as one that cannot interact with something physical. Then, of course, interaction is impossible by definition.

But this definition does great injustice to the dualistic position by unnecessarily requiring the dualists' concept of mind to emphasize the absence of physical characteristics, while ignoring more general, basic 
aspects of dualism. This state of affairs owes its origins to the philosophical works of René Descartes, who first delineated a specific conception of interactionism, and to the responses to his work.

Descartes' (1641/1986) Meditations on First Philosophy changed the philosophical landscape on the mind/body issue by proposing explicit and radical differences between mind and body, thus starting an enduring controversy. Therefore, in working towards a reasonable definition of dualism, it is vital first to understand Descartes' position, which I reviewed in detail in Part II. To summarize, Descartes' view of the nonphysical mind included the following properties: (1) the mind can survive the death of the body; (2) mind is a thinking substance; (3) the mind is not extended, and so does not occupy space to the exclusion of other objects, and does not have shape, position, or motion; and (4) the mind is physically indivisible.

These are indeed radical distinctions between mind and body, and during the intervening centuries Descartes' distinctions have been sharpened by materialists to consist of the following description of the dualistic position: the nonphysical mind has neither matter nor energy, is not localizable, is not extended in space, has no physical properties whatsoever, and works independently of the brain (Bunge, 1980). This is even more radical than Descartes' own proposal.

Must modern interactionist theories be constrained by Descartes' original vision and the baggage subsequently strapped onto it? Why should we expect Descartes' view, proposed at the very beginning of the scientific age, to have sprung forth full-blown and irrefutable? Indeed, Descartes was responsible for the origination of an early form of the scientific method and for the invention of analytic geometry, both of which were required for the later flowering of science (Viney, 1993). From his position in history, then, it would have been impossible for Descartes to propose a complete, scientific theory of interaction.

One should therefore ask: which of Descartes' stated mental properties are truly needed for a definition of dualism, and which are unnecessary? It seems clear that the concept of mind as a thinking substance that survives bodily death and that is physically indivisible is fundamental to the dualist view. But what of the concept of extension and its properties? Since no one has ever seen the nonphysical mind or measured its properties in any way, it is reasonable to suggest that this substance does not share the property of the exclusionary occupation of space displayed by matter. But if the mental substance is in fact a substance in its own right, there should be no a priori reason that it could not exist in space and time, have shape, or have location. 
A consistent and useful definition of dualism, then, should not be constrained to focus on properties (and particularly on extension) but rather should attend to the more fundamental aspects of the mind/body distinction. I propose that a reasonable definition of dualism should focus on three highly related but somewhat different concepts: reduction, substance, and survival. First, one may ask whether mind can be reduced to brain. That is, can consciousness be reduced to a product of brain functioning, or is consciousness itself irreducible? Second, are humans composed of a single substance, matter, or might we be a combination of matter with some other, currently unknown, building block? Third, is what we know as death the complete termination of our existence, or is survival of death in some nonphysical form possible?

Obviously, materialism has ready answers to the three questions. First, consciousness is the direct result of the living brain, and has no other source. Second, the universe as a whole, and human beings in particular, are composed only of matter, existing together with energy. And third, death is the termination of our bodily processes, and thus the end of our consciousness. Interactionism, of course, answers differently on all three counts, embracing a position of irreducible consciousness, two distinct substances interacting during physical life, and the mind's survival of death. In this conception of dualism, the mind could conceivably have certain physical properties, yet the theory would still be dualistic; in this context, "nonphysical" means nonmaterial, but not insubstantial. Thus, this triangular definition of dualism clearly distinguishes between materialist and interactionist perspectives without undue focus on physical properties.

\section{Matter as an Example of Substance}

One might then ask as to the nature and definition of "nonmaterial substance." In particular, one could challenge the idea of essence, suggesting that positing such a substance is arbitrary and unprecedented. But in fact we need look no further than general relativity for insight into the possible nature of substances other than matter.

The theory of general relativity (Einstein, 1961; Hawking, 1988; Misner, Thorne and Wheeler, 1973; Thorne, 1994) has caused a revolution in the scientific view of the nature of matter, energy, space, and time. (I discussed space and time in the context of the theory of essence in Part I.) Just as space and time are closely related, so relativity demonstrates that matter and energy are also intimately intertwined. Relativity provides a mathematical relationship between the 
two: matter and energy are linearly related. The energy contained in a given amount of matter is the mass multiplied by the speed of light squared. From an energetic point of view, this relationship provides a basic definition of matter. Since the speed of light is a very large number, a small amount of matter is equivalent to a huge amount of energy. For example, a few pounds of the proper isotope of uranium are sufficient to cause an atomic blast that can level a large city.

One can thus view matter as a form of condensed energy, which can be released under the proper conditions. A corollary of this principle is that the substance called matter has a certain energy density, given by the linear relationship discussed above. Consider the possibility, then, that other substances, representing alternative relationships with energy, are possible. Specifically, one can propose that an entire series of substances is theoretically possible, with the substances differing from each other in their energy densities. The definition of substance, in this view, would therefore be any form of condensed energy.

For clarity, it is helpful to consider a specific example. Matter could be viewed as a particular case of a general substance/energy (E) relationship, to wit:

$$
\mathrm{E}=\mathrm{S}_{\mathrm{i}} \mathrm{c}^{\mathrm{i}}
$$

In the case of matter (substance $S_{2}$ ), the proportionality constant is $\mathrm{c}^{2}$; each possible substance would be represented by a distinct value of the index $i$. Essence, for example, could be represented by substance $S_{1}$. In that example, essence would be related to energy through the proportionality constant $\mathrm{c}$, and essence would therefore be much less energy-rich than matter.

The energy-density difference between $S_{1}$ and $S_{2}$ would be much more fundamental than, say, the observed changes in the density of matter during phase changes, such as liquid water turning to water vapor. The present concept of substance would require that nonmaterial substances display basic differences from matter in the way those substances are constructed from energy, and one could accordingly expect important property differences among the substances $\mathrm{S}_{\mathrm{i}}$. It is not unreasonable, for instance, to propose that substances $S_{1}$ and $S_{2}$, being very different in energy density, could simultaneously occupy the same three-dimensional space. It is important to emphasize that $S_{1}$ is not merely some less-dense form of matter, but is an entirely different substance. 
A useful way to conceptualize the potential properties of $S_{1}$ is to examine the series $S_{2}, S_{1}, S_{0}$. Substance $S_{0}$ would be identical to energy itself. $S_{1}$ (essence) would lie between energy and matter $\left(S_{2}\right)$ in terms of energy density and physical properties. Thus if $S_{1}$ could be examined with traditional scientific instruments, it would appear to be much more similar to energy than is matter. This idea receives support from the NDE literature: several of Raymond Moody's (1975) research participants described their disembodied selves in terms of energy (Arnette, 1995a). For example, one NDEr described himself as "a little ball of energy" (Moody, 1975, p. 50).

Although the various $S_{i}$ would exhibit considerable differences, they could also conceivably share some properties. Again, consider matter: despite the fact that energy undergoes a radical transformation to become matter, signatures of that energy remain in the material form. For example, on the subatomic scale, electrons display both wave-like and particle-like properties (Eyring, Walter and Kimball, 1944; Landau and Lifshitz, 1977). Another signature is the electric charge associated with elementary particles-protons being positively charged and electrons being negatively charged-and the electromagnetic fields generated as a result of those charges (Halliday and Resnick, 1970). It is then fair to propose that other substances could also retain energetic signatures analogous to, or even perhaps identical to, electric charge. Supposing that the fields generated by substances $S_{1}$ and $S_{2}$ are similar in nature, these fields would form the basis of body/essence binding and interaction, as I described in Part II.

A final point concerns gravity (Krishnan, 1999). In Part I, I suggested that the essence is not constrained by the spacetime of this universe, thus enabling the essence to observe and enter wormholes that are otherwise invisible; and that because gravity is the warping of spacetime by mass, the essence would neither cause nor be affected by gravity. It is therefore quite feasible for the essence to possess an electric field but be unaffected by gravity.

Suppose, for the sake of argument, that substance $S_{1}$ (and therefore the essence) is for some reason subject to the force of gravity. It is important to remember that gravitation is by many orders of magnitude the weakest force known in nature (Halliday and Resnick, 1970), and would be expected to have a negligible effect on $S_{1}$. For example, the massless photon, the smallest unit of energy, is correctly predicted by relativity theory to be influenceable by gravity, but the amount of mass needed to observe this effect experimentally is literally of stellar proportions (Hawking, 1988; Thorne, 1994). $S_{1}$, lying between $S_{0}$ and $S_{2}$ in 
its properties, would be more easily influenced than photons, but certainly much less subject to gravity than is matter. Thus the influence, if any, of gravity on the essence should be unobservable in a terrestrial environment, as many NDErs have reported (Arnette, 1992; Moody, 1975; Ring, 1980).

The preceding discussion shows that relativity theory provides a matter/energy relationship that can serve as a prototype for the definition of other possible substances, and for hypotheses concerning the properties of these substances. The concept of substances with energy densities different from matter is not unprecedented. Kip Thorne (1994), a prominent cosmological theorist, has noted that relativity theory posits that "exotic material," with a negative energy density relative to an observer traveling near the speed of light, could conceivably be used to construct artificial wormholes, and that this material actually exists in the vicinity of black holes.

\section{Neuroanatomical Aspects of Interactionism}

Now the task remains to go beyond the general notions of essence/ brain interaction given in Part II and propose a more detailed description of interaction at the anatomical and microscopic levels. As mentioned above, the theory of essence conceives of mind as being formed by and consisting of the overlapping electromagnetic fields of the brain and essence, with the mutual influences of these fields providing the basis for reciprocal causation between brain and essence (Arnette, 1995a). Thus the brain is far from being irrelevant to interactionism, as some have implied (Bunge, 1980; Dennett, 1992; Gómez-Jeria and MadridAliste, 1996). Instead, the brain is the crucial interface between the essence and the physical world (Arnette, 1995a). But a complete interactionist theory must go further and explain how the essence and brain interact on a detailed level. And such a discussion must first focus on the anatomy of this interaction.

For the present purpose, I will present a brief general overview of the brain and its functioning. A reasonable perspective in this regard is provided by Aleksandr Luria's (1973) model of brain structure and function. While it is a fallacy to assume that any parts of the brain function independently, it is nonetheless true that there is considerable localization of function. Luria's model recognizes the interdependence of the various brain regions but also organizes brain functions according to structure. Luria's conceptualization divides the brain into three principal functional units, as follows. 
Unit I interacts with the other two units to regulate cortical and emotional arousal, which arises from external stimuli, metabolic processes, intentions, and plans. Unit $I$ is particularly involved in attention and attentional shifts. The anatomy of this unit includes the reticular and limbic systems, the thalamic nuclei, and the anterior frontal lobes. Unit I, except for the frontal lobes, constitutes the phylogenetically oldest parts of the mammalian brain.

Unit II consists of the cortex posterior to the central sulcus, and is responsible for obtaining, processing, and storing information. This unit is divided into three cortical zones, each with somewhat different functions. The primary zone receives sensory signals from peripheral brain regions; the secondary zone processes this information and forms perceptions; the tertiary zone performs complex information processing, involving the coordination and simultaneous use of many cortical areas, and stores information.

Unit III consists of the cortex anterior to the central sulcus, and serves to program, regulate, and verify information. This unit is also divided into three zones. The tertiary zone monitors behavior by comparing incoming and outgoing information, formulates intentions and plans, and controls behavior; the secondary zone prepares motor programs; the primary zone executes the motor programs by sending the appropriate signals to skeletal muscles.

In the present context, the regions of greatest interest are the cortical zones, especially the tertiary zones of Units II (temporal lobe, temporo-occipital region, and superior and inferior parietal lobes) and III (prefrontal lobes). These regions carry out the highest of the brain's functions, executing complex mental activities and coordinating the functions of other cortical regions. It is in these areas that information enters, judgments are made, and decisions for action go out to the body.

Luria (1973) noted that the tertiary structures of Unit II are uniquely human. These zones provide for "the transition from direct, visually represented synthesis to the level of symbolic processes" so that "the tertiary zones of the posterior cortical region play an essential role in the conversion of concrete perception into abstract thinking" (Luria, 1973, p. 74; emphasis is the author's). The tertiary zones of Unit III make use of the results of Unit II's operation. Again quoting Luria:

[W]hereas in the second, afferent system of the brain [Unit II] the processes go from the primary to the secondary and tertiary zones; in the third, efferent system [Unit III] the processes run in a descending direction, starting at the highest levels of the tertiary and secondary zones, where the motor plans and programmes are formed, and then 
passing through the structures of the primary motor area, which sends the prepared motor impulses to the periphery. (1973, pp. 82-83)

Luria then pointed out that Unit II consists entirely of efferent neurons and possesses modally specific individual analysis zones, while Unit III does not display such specific organization and consists completely of afferent, motor-type neurons. A final point from Luria's work is that humans posses the most highly developed frontal lobes in the animal kingdom, and

that is why in man, through the progressive corticalization of functions, processes of programming, regulation and verification of conscious activity are dependent to a far greater extent on the prefrontal parts of the brain than the processes of regulation of behaviour are in animals. (Luria, 1973, p. 93)

The crucial role of the prefrontal lobes in the qualities that appear to be unique to humans (such as planning, judgment, and abstract and symbolic thinking) has been demonstrated by studies of the behavior of individuals with focal damage to, removal of, or disease processes within their prefrontal regions (Luria, 1973; Pearlman and Collins, 1990).

Even with all the progress made to date in neuropsychology, we still do not understand how these highest cortical regions execute their functions (Collins, 1990). We do know that these regions are multiply connected to and, to a large extent, oversee the involvement of other cortical and lower brain regions (Collins, 1990; Luria, 1973), but how this is all accomplished is something of a mystery (Penfield, 1975). The theory of essence does not attempt a complete answer to this question, but does hold that the tertiary regions of Units II and III in Luria's model are crucial to our functioning as humans because these are the interfacial regions between the essence (the seat of consciousness) and the remainder of the brain.

Specifically, the theory posits that the essence is superimposed on the entire central nervous system (CNS). By means of the electromagnetic interaction mechanism outlined above and explained in more detail below, the essence potentially can gather information from anywhere in the CNS; but the most integrated, organized, complete information is available from the efferent neurons in the Unit II tertiary areas. Likewise, there must be an entry point in the biological system for information (commands) from the essence concerning the actions of the body; the afferent neurons of the Unit III tertiary regions provide this opportunity. Thus, the essence fills a gap, as it were, between the "upstream" functions of Unit II and the "downstream" functions of Unit III. 
The essence occupies a role that mediates between the tertiary areas of these two units - coordinating them, bridging them, and interacting with them to receive, analyze, and provide information. Thus, these areas (and especially the prefrontal lobes) are necessary for us to be human in all senses of that word, but are not sufficient: the essence is required as well.

Regarding the evolution of human consciousness (Gómez-Jeria and Madrid-Aliste, 1996), there is absolutely nothing in the theory of essence that is inconsistent with evolutionary principles. The essence works in concert with the brain, and the expression of the essence (and therefore consciousness) through the brain is enhanced as the sophistication of the interface increases. If consciousness offers a survival advantage, which certainly seems plausible, then the presence of the essence would be expected to drive the evolutionary process to produce an increasingly complex brain with ever-growing interfacial regions reflecting more specialization for the purpose of linking the essence with the body. This picture is in complete harmony with Luria's (1973) comments quoted above and with many of the concepts discussed by Gómez-Jeria and Madrid-Aliste (1996).

As to the question of when the essence arose during the course of evolution (Gómez-Jeria and Madrid-Aliste, 1996), there is no reason why the existence of the essence should depend in any way on a terrestrial evolutionary process. It is in fact highly problematic to suggest that substance $S_{1}$ evolved from, or was in some other manner produced by, substance $S_{2}$ within earth's environment. As I discussed in Part I, the theory of essence proposes that wormholes provide passageways to other universes. During an extended NDE, the dissociated essence may travel through a wormhole to another universe. Often, NDErs report encounters with deceased individuals the NDEr had known during physical life (Moody, 1975; Ring, 1980). The data provided by Kenneth Ring (1984) in his discussion of extended experiences and by Morse (1983) in his report of a child's NDE also indicate that NDErs sometimes meet individuals waiting to return to physical life. These data imply that the individual essence has a continuous existence, both existing before and continuing beyond terrestrial life.

The theory of essence thus views wormholes as two-way portals. The essence comes through a wormhole into this universe, our familiar fourdimensional spacetime, in order to join with a physical body and live a physical life; and when that life is over, the essence returns to its original spacetime through a wormhole. The essence did not "evolve" in the context of this universe. Gómez-Jeria and Madrid-Aliste (1996, p. 267) have already objected to this type of reasoning, equating the 
concept of "other dimensions" with pseudoscience. However, modern theoretical cosmology treats the existence of higher dimensions as fact, and the existence of other universes (that is, other spacetimes) as a strong possibility (Halpern, 1992; Hawking, 1988; Misner, Thorne and Wheeler, 1973; Thorne, 1994; Wolf, 1988). Thus, the theory of essence, far from being unfriendly to biological science, instead incorporates it logically while simultaneously integrating the NDE data and the results of general relativity.

\section{Neurophysiological Aspects of Interactionism}

Having proposed specific anatomical regions for maximal essence/ brain interaction, I next address interaction at the microscopic, or neuronal, level. Richard Taylor (1992) has phrased this issue as: how can an idea fire a neuron, and conversely, how can a neuron generate an idea? Indeed, this appears to be a fundamental question for any mind/body theory. The response to this question begins with a description and analysis of the neural firing process.

\section{The Mechanism of Neural Firing}

The firing process (Carlson, 1986; Stryer, 1988) of any given neuron begins with the transmission of electrochemical signals, known as action potentials, to that neuron from other neurons. Consider as an example the case of the firing of unmyelinated, excitatory neurons. Neuron A interfaces with neuron B at the synapse, where A's terminal button lies across the synaptic gap from B's post-synaptic membrane. The action potential migrates down A's axon to the terminal button by a process called orthodromic conduction, where it causes the release of neurotransmitter molecules into the synaptic gap. The neurotransmitters then diffuse across the gap, and bind to receptors on the post-synaptic membrane.

Binding of the receptors by neurotransmitters opens ion gates in the membrane, thus allowing the passage of sodium ions from the gap, through the membrane, and into the cytoplasm. This influx of sodium ions lowers the difference in electric potential energy between the interior and exterior of the neuron's cell body. When the potential difference moves from its resting value of -70 millivolts to less than -65 millivolts, the threshold value, the cell is depolarized and the excitatory post-synaptic potential is formed.

This excitatory post-synaptic potential may be sufficiently large that the potential at the axon hillock, where the cell body and axon meet, is 
driven past the threshold value and the neuron fires. However, if the excitatory post-synaptic potential is below that threshold value, it dissipates without effect. Thirdly, the excitatory post-synaptic potential may be summed spatially or temporally with other excitatory postsynaptic potentials and/or inhibitory post-synaptic potentials (generated by inhibitory neurons); again, when the potential at the hillock passes threshold, the neuron fires.

At the hillock, when the potential passes threshold, ion gates are again opened and sodium ions are allowed to pass through the membrane. The potential difference increases as the sodium migration continues, reaching equilibrium at +50 millivolts. The +120 millivolt change (from -70 to +50 millivolts) is the action potential. As the sodium ions enter the hillock, potassium ions are electrostatically forced out of the axon ahead of the sodium influx. This reciprocal ion flow sets up eddy currents that spread in all directions from the hillock.

Those currents that are transmitted to the cell body eventually die out. Those transmitted down the axon are maintained by orthodromic conduction, in which the sodium/potassium ion exchange is replicated at regular intervals down the axon. The action potential generates its own replication by opening sodium channels in the neighboring segment of axon. Thus the action potential is transmitted to the terminal button, where the firing process begins again.

In the wake of the action potential, work must be done to return that segment of the axon to its original status so that it can conduct the next electric field pulse. Sodium ions must be pumped out of the cell, across the cell membrane, and back into the extracellular fluid. Potassium ions must be pumped in the reverse direction. This ionic rearrangement opposes the natural direction of diffusive flow, thus requiring the input of energy. Once the original ionic distributions are regained, this segment of the axon (or of the post-synaptic membrane) is ready to fire again. It is, in a sense, reloaded and awaits retriggering.

Relevant to Cornman's (1981) objections cited above and to the essence/brain interaction mechanism, there are several important points to be made from the preceding discussion. First, the potential at the axon hillock is the sole criterion for neural firing. Second, the energy associated with neural firing is expended in advance of the actual firing of the neuron, when the sodium and potassium ions are transported against the diffusive flow.

Third, it is the action potential, an electric field pulse, that is propagated along neural pathways; no material particles are transmitted along the axon. Although ions are transported across membranes, the action potential's direction of propagation is perpendicular to the 
direction of ionic motion. Fourth, when an axon branches, the action potential sets up eddy currents in, and is propagated along, all branches. And fifth, the process at the synapse involves the release, diffusion, and binding of neurotransmitters, which are chemical processes; the meaning of "the resistance at the synapse" is thus not clear, and any such resistance would appear to be irrelevant to the process.

In light of these points, Cornman's (1981) misconceptions become clear. First, no redirection of neural impulses occurs; the impulses are transmitted along all axons at all branch points. Second, since the action potential is a field without mass, there is no linear momentum to be conserved. The laws of electromagnetism, not the laws of classical mechanics, apply to neural firing. Third, the energy of a firing neuron is supplied biologically in advance of the firing, and so a nonphysical mind (that is, the essence) would not need to supply this energy to the neuron. Additionally, the preceding discussion reveals Broad's (1925) misconception: the "resistance" at the synapse is an illdefined concept that is not relevant to the neural firing process, and thus provides no apparent way for the mind (essence) to influence the brain.

\section{A Mechanism of Essence/Neuron Interaction}

This examination of the mechanism of neural firing serves both to dispel misconceptions about the process and to reveal the mode in which the essence can interact with the brain. The relevant conclusion here is that the potential at the axon hillock is the sole criterion for neural firing. Because of this fact, the firing of a neuron can be accomplished without the depolarization of the cell body.

Such a conclusion may seem startling, but in fact has already been experimentally demonstrated. In his surgical experiments with epileptic patients, neuroscientist Wilder Penfield (1955, 1975; Penfield and Rasmussen, 1950) applied electrical stimulation to the exposed neurons of the temporal region of the brain while patients were fully conscious. The stimulation resulted in the patients' experiencing full, vivid, and accurate memories from both the recent and distant past. These experiments helped reveal the function of various brain regions, and also show that imposed, nonbiological fields are capable of firing neurons by pushing the potential at the hillock beyond the threshold value.

The proposed interaction mechanism, then, between essence and the brain's neurons is this: the essence and brain occupy the same three-dimensional space and are bound together by electromagnetic 
forces. Essence and brain interface with each other through the interpenetration and reciprocal causal influence of their respective electromagnetic fields, which vary in time. A thought, idea, or mental image, the basic unit of our conscious experience, is defined as the threedimensional configuration of the essence electric field at a given point in time. During the essential thinking process, the essence field fluctuates and changes in configuration, and these fluctuations are sensed at the neurons' axon hillocks; when the essence field drives the hillock potential past threshold, the neuron fires. This is how an idea can fire a neuron.

Conversely, neurons affect the essence by way of a complementary electromagnetic mechanism. When signals from the body are conducted to the brain, these signals are sensed by the essence as fluctuations in the brain field. These fluctuations contain information collected from the various senses and the central nervous system. The essence receives this information through the interaction of essence and brain fields, especially in the interfacial regions (the tertiary areas of Luria's Units II and III). Thus neurons cannot create an idea, for the essence drives thought. But neurons can, through this mechanism, contribute information that helps form thoughts, ideas, and images.

The picture that emerges from this proposed mechanism is one of constant interaction and communication between essence and brain through the fluctuations in the electromagnetic interface. Signals generated by the brain and nervous system are continually sensed by the essence, which in turn continuously influences the brain by virtue of the essential thinking process and the associated field fluctuations. It is not clear whether energy must move between brain and essence during these interactions, but it is probable that very small amounts of energy do move. If that is the case, then there is clearly an energy exchange process, as I implied in the dipolar relaxation analogy discussed in Part II.

Since the two fields, and thus the two substances, constantly intervene on each other, it is therefore reasonable to propose that the two substances are in a dynamic equilibrium. In this picture, energy is always moving from essence to brain, and from brain to essence. The flow is balanced, such that the net exchange is zero over time even though energy is constantly flowing. This is the case with chemical systems at equilibrium, in which individual molecules are constantly decomposing but are also continuously being regenerated, and with physical systems in thermal equilibrium, in which thermal energy is constantly exchanged but with no net transfer of heat (Levine, 1978). 


\section{Implications for the Multiplicity Problem}

The final task of the present work is to address several sources of NDE-like experiences, proposed by materialists as causes of and explanations for the NDE. The goal is to show that these sources have a common mechanism of action that is predicted by the anatomical and physiological aspects of the theory of essence, thereby demonstrating that the theory has significant predictive power and the ability to unite many seemingly diverse phenomena.

As mentioned above, Part II of this model developed the idea that the attractive force between body and essence was analogous to, and could be modeled as, the interaction between two electric dipoles (Arnette, 1995a). A dipole is defined as a positive charge held at a fixed distance from an equal negative charge, and represents the simplest distribution of charge separation. Part II discussed this model in the context of binding the body with the essence, but such a model may be used on any size scale to describe electromagnetic interactions. In fact, it is common in the field of neurology to use dipole approximations in modeling cortical pathophysiology (Baumgartner, Sutherling, Di and Barth, 1991; Sutherling, Crandall, Levesque, Darcey and Barth, 1992). In this paper I used the dipole model to describe the interactions of the essence with the interfacial regions of the brain, the tertiary areas of Luria's Units II and III.

Because both the essence and brain electric fields change with time, the simplest model of their interaction would employ two oscillating dipoles, each with its own frequency of oscillation. An attractive force between these dipoles results when the two oscillation frequencies are matched - that is, when the dipoles are tuned to each other (Arnette, 1995a). The physics of even a simple system such as this one is highly complex and well beyond the scope of this article; I refer the reader to Paul Lorrain and Dale Corson (1970, pp. 595-620) for a detailed discussion.

The important point for the theory of essence is that the electric field intensity, and therefore the lines of force, between the oscillating dipoles depend on the relative values of the oscillation frequencies. When the oscillations are in phase, the dipoles tuned to each other, the dipolar fields interfere constructively and the force is attractive; as the dipoles move out of phase, constructive interference is replaced by destructive interference and the attraction dissipates.

In terms of the essence/body system, this tuning mechanism means that deviations of either the essence or body oscillation frequencies from their matched values can result in partial or complete essence/body 
dissociation. From the bodily side, partial dissociation can occur when, for example, the firing rates of the neurons in the interfacial regions of the brain are significantly altered from their normal ranges. Complete dissociation can occur in cases such as grave illness, severe physical injury, or physical death, when the entire body's electrochemical system is nearly or completely shut down.

Consequently, the theory of essence predicts that factors affecting neural firing rates in the tertiary areas of Units II and III or in the entire body can lead to NDEs or NDE-like experiences. Further, the theory predicts that some sources of frequency shifts causing partial dissociation, such as drugs or temporal lobe epilepsy, will have additional effects that lead to deviations from the standard NDE phenomena. This is because there is still a connection between the essence and the brain, which continues to function and is influenced in multiple ways by the source of the frequency shift.

The theory thus leads to predictions that are empirically falsifiable, in the sense that Karl Popper (1959) has used this term. Opportunities to test these predictions arise from the various factors suggested from within the materialist paradigm for explanations of the NDE. Several of these factors were mentioned in connection with the models of Morse and colleagues and Saavedra-Aguilar and Gómez-Jeria discussed above, including electrical stimulation, hypoxia, drugs, temporal lobe disturbances, psychological stress, and the action of neurotransmitters.

Sabom (1982) reviewed many of these factors, and found each of them lacking in explanatory power due to mismatches between the symptoms caused by each factor and the core phenomena of the NDE. Yet, several of the factors do have some area of commonality with the NDE, often in the form of symptoms of dissociation: feelings of detachment from one's body, emotions, and/or thought processes; observing one's body from outside it; and distortions in the perception of time and/or space (Steinberg, 1994). This paradoxical situation can be resolved by considering the physiological effects of these factors in the context of the theory of essence.

\section{Endogenous Electrical Stimulation}

Endogenous electrical stimulation of the brain's interfacial regions accompanies complex partial seizures, formerly referred to as temporal lobe epilepsy. Eric Lothman and Robert Collins (1990) wrote that "[i]n every instance seizures arise because of an abnormal, excessive, paroxysmal, synchronous discharge in a population of neurons," with 
these discharges being "both transient and readily differentiated from the normal background activity of the brain, features encompassed by the term paroxysmal" (p. 276). These seizures affect both the temporal and prefrontal lobes (Unit II and III tertiary areas, respectively).

Complex partial seizures therefore meet the requirements of the theory of essence for inducing partial essence/body dissociation. The clinical literature supports this theoretical prediction. Complex partial seizures and similar disorders can cause dissociative symptoms (Loewenstein and Putnam, 1988) that differ significantly in intensity and frequency from those in normal populations (Persinger and Makarec, 1993); OBE-like experiences (Persinger, 1995); and autoscopic phenomena (seeing one's own body) similar to NDEs (Devinsky, Feldmann, Burrowes, and Bromfield, 1989). Also in accordance with the prediction, there are significant differences between temporal lobe epilepsy and dissociation (Brown, 1994) or NDEs (Sabom, 1982).

\section{Exogenous Electrical Stimulation}

Exogenous electrical stimulation of interfacial regions also has effects that are consistent with the theory's predictions. I cited Penfield's $(1955,1975)$ experiments with temporal lobe stimulation above as evidence that imposed electric fields could fire neurons. In fact, more than memories could be stimulated by this method: "[the patient] may feel as though he were far away and yet can perceive the scene, may seem to see himself and know what is happening to his body, as though he were a secondary observer" (Penfield and Rasmussen, 1950, p. 173).

More recently, it was reported on the television show Turning Point (Donvan, 1994) that neurologist William Sutherling has verified Penfield's observations, employing subdural platinum-iridium electrodes to map the brains of epileptic patients prior to surgery. In one of his patients, for example, electrical charge was delivered to the temporoparietal lobe (a tertiary area of Unit II), resulting in the patient reporting an OBE. And finally, electroconvulsive therapy has been shown in some cases to cause autoscopic phenomena (Devinsky, Feldmann, Burrowes, and Bromfield, 1989) and in at least one case even a fullblown NDE (Floyd, 1996).

\section{Psychoactive Drug Action}

Several authors have claimed that the features of the NDE can be reproduced by the ingestion of psychoactive drugs. Two drugs frequently mentioned in this regard are the anesthetic ketamine (Collier, 1972; Jansen, 1990, 1997; Rogo, 1984; Morse, Venecia and Milstein, 1989) and 
the hallucinogen lysergic acid diethylamide (LSD) (Grof and Halifax, 1977; Morse, Venecia and Milstein, 1989). Sabom (1982) has pointed out that the reaction to such drugs is highly idiosyncratic, in sharp distinction to the core NDE, and that many of the drugs' effects have nothing to do with the NDE.

It nevertheless appears that what similarities do exist between the $\mathrm{NDE}$ and drug action are strong enough that theorists continue to make a connection (Jansen, 1990, 1997; Morse, Venecia and Milstein, 1989). The basic line of reasoning is this: drugs bind to certain receptors in the brain, which then trigger NDE-like events; this action mimics the effects of endogenous neuropeptides and neurotransmitters that cause similar effects in times of intense stress, pain, and physical injury (Jansen, 1990, 1997; Morse, Venecia and Milstein, 1989; SaavedraAguilar and Gómez-Jeria, 1989).

From the perspective of the theory of essence, similarities between drug action and the NDE are due to a partial essence/brain dissociation caused by the alteration of neural firing rates in the brain's interfacial regions. That the dissociation is partial and that drugs have effects in other parts of the brain account for those drug effects that are idiosyncratic and unlike NDEs.

Consider first the physiological effects of LSD. This drug is an antagonist of the neurotransmitter serotonin (Carlson, 1986; Pierce and Peroutka, 1990), affecting prefrontal cortical serotonergic function (Breier, 1995) and serotonergic cortical function in general (Aghajanian, 1994). An antagonist, by definition, inhibits or blocks the action of a neurotransmitter (Carlson, 1986) and thereby alters the firing rates of the associated neurons from their normal values.

Similarly, ketamine is an antagonist of $\mathrm{N}$-methyl-D-aspartate (NMDA) (Jansen, 1990, 1997; Lahti, Koffel, LaPorte and Tamminga, 1995) and acts on thalamocortical pathways (Saletu, 1987) as well as through cerebral mechanisms (Gordh, Karlsten and Kristensen, 1995). It is significant that NMDA receptors have also been implicated in epileptic seizures (Lothman and Collins, 1990). Thus both LSD and ketamine fulfil the requirements of the theory of essence for partial dissociation of the essence from the brain.

\section{Psychogenic Frequency Shifts}

Individuals faced with life-threatening situations have reported dissociative symptoms with similarities to NDEs (Noyes and Kletti, 1976a, 1976b). Even though Russell Noyes (1978), a major researcher in this area, has stated that these experiences differ significantly from those 
of NDErs who are physically near death, the notion of psychological stress continues to be highlighted as a key causative factor for NDEs (Ferris, 1991; Morse, Venecia and Milstein, 1989; Saavedra-Aguilar and Gómez-Jeria, 1989).

In this regard, the theory of essence holds that changes in the dipolar oscillation frequencies of both the body and the essence can have psychological, rather than physiological, origins. Consider the case of a life-threatening situation or otherwise highly stressful event. The endangered individual first makes a cognitive appraisal that establishes the presence of a threat (Folkman, Lazarus, Gruen and DeLongis, 1986; Lazarus and Folkman, 1984). The perception of a threat can in turn initiate the "fight-or-flight" response, the activation of the sympathetic nervous system (Rice, 1987), which has strong effects throughout the body. This may occur even with the purely mental re-experiencing of the event.

Sympathetic nervous system activation at a sufficiently high level can potentially alter the brain's as well as the entire body's oscillation frequency, which could consequently induce essence/body dissociation. It is in accordance with the theory, then, that dissociative symptoms are associated with posttraumatic stress disorder (Brown, 1994; Brende and Benedict, 1980; Steinberg, 1994), incest (Ellenson, 1985; Greer, 1994; Kluft, 1990; Lester, 1989; Maltz, 1988; Silon, 1992), and physical abuse (Carlson, 1994). Additionally, the essence may itself shift in frequency due to the perception of a threat. This could be viewed as a defensive effort by the essence to avoid physical and/or emotional pain, a hypothesis that is consistent with the general view that dissociative symptoms in incest survivors serve an adaptive and defensive function (Greer, 1994; Lester, 1989; Silon, 1992).

A final psychogenic factor, which has not been cited directly in the literature as a cause of NDEs, is that of meditative states. According to the present theory, shifts in the essence frequency, probably with concomitant shifts in the body's frequency, can be induced consciously and willfully through meditation and related techniques. This would probably require training and practice, and may bear some relation to Eastern philosophical and religious traditions. It has in fact been shown that meditative states can result in altered states of consciousness (Khatami, 1978) and OBEs (Dane, 1975-76; Grosso, 1976).

\section{Shifts in the Equilibrium of Glucose Oxidation}

A final source of frequency changes lies in the most vital chemical reaction occurring in the brain: the oxidation of glucose. The brain 
depends completely on the oxidation of glucose for all its energy needs (Powers, 1990); glucose and oxygen react to form products, including water and carbon dioxide, and energy is released in the process. As with any chemical reaction (Levine, 1978), glucose oxidation is an equilibrium between reactants and products. Thus the reaction can be slowed or stopped by an increase in the concentration of the products, such as carbon dioxide, or a decrease in the concentration of reactants, such as oxygen. As the reaction slows, so necessarily must the rate of neural firing.

The theory of essence posits that a decrease in neural firing causes a shift to lower dipolar oscillation rates in the brain and/or body, which at some point must lead to essence/body dissociation. Certainly at the point of physical death, which could be defined as the complete cessation of the glucose oxidation reaction, dissociation must begin. Short of death, other physical conditions such as severe physical injury or illness can effect at least a partial dissociation.

Again, the literature supports this contention of the theory. Autoscopic phenomena have been associated with toxemia of pregnancy and severe infection (Devinsky, Feldmann, Burrowes and Bromfield, 1989). Dissociative symptoms can be associated with physical illness (Ross, 1994). Full-blown NDEs are very often triggered by cardiac arrest (Sabom, 1982), drowning, physical injury, and illness (Moody, 1975; Ring, 1980). And hypoxia, the lack of oxygen, and hypercapnia, an excess of carbon dioxide, have been cited as integral factors in materialistic theories of the NDE (Morse, Venecia and Milstein, 1989; SaavedraAguilar and Gómez-Jeria, 1989).

Perhaps most telling are the results of a technique known as carbon dioxide therapy (Meduna, 1950), in which carbon dioxide levels in the blood of subjects was intentionally elevated. Of all the suggested factors for NDE causation reviewed by Sabom (1982), this was the sole factor having effects closely resembling the NDE. The strong similarity between the NDE and the effects of carbon dioxide therapy led Sabom (1982) to propose that the build-up of carbon dioxide in the brain was a trigger for the NDE. From the perspective of the theory of essence, this proposal makes chemical and electromagnetic sense and is eminently reasonable.

\section{Conclusion}

The preceding discussion demonstrates that the theory of essence has strong predictive validity, integrating the effects of four classes 
of factors (internal and external electric fields, psychoactive drug action, psychological factors, and glucose oxidation equilibrium factors) into a single mechanism that accurately predicts effects along a continuum from psychiatric dissociative symptoms through OBEs to complete NDEs. While the theory does not treat the temporal lobe as the "seat of the soul," as Morse has suggested, it does present the tertiary areas of the brain as, in some sense, the "anchor of the essence."

The present work has shown that interactionism is a viable scientific concept, once liberated from its historical shackles. The triangular definition of dualism, in terms of substance, reduction, and survival, removes the focus on physical properties or the lack thereof, and frees dualists to employ the results of more than three centuries of scientific inquiry. In so doing, the interactionist discovers that the physical and nonphysical can in fact be compatible and complementary, rather than contradictory. The resulting theory corrects the misconceptions of dualism's critics, while supplying a reasonable picture of dualistic interactionism. Of course, this picture generates many new questions, and many old ones remain. But this is axiomatic in science, and provides directions for future inquiry.

\section{References}

Aghajanian, G. (1994). Serotonin and the action of LSD in the brain. Psychiatric Annals, $24,137-141$.

Arnette, J. K. (1981). Rotational relaxation of a dipole in two dimensions. Unpublished doctoral dissertation, Florida State University, Tallahassee, FL. (Abstracted in Dissertation Abstracts International, 42, 2388B.)

Arnette, J. K. (1992). On the mind/body problem: The theory of essence. Journal of NearDeath Studies, 11, 5-18.

Arnette, J. K. (1994). On the mind/body problem: Kenneth Arnette responds. Journal of Near-Death Studies, 13, 139-141.

Arnette, J. K. (1995a). The theory of essence. II. An electromagnetic-quantum mechanical model of interactionism. Journal of Near-Death Studies, 14, 7799.

Arnette, J. K. (1995b). A critique of pure materialism. Unpublished master's thesis, Colorado State University, Ft. Collins, CO.

Baumgartner, C., Sutherling, W., Di, S., and Barth, D. (1991). Spatiotemporal modeling of cerebral evoked magnetic fields to median nerve stimulation. Electroencephalography and Clinical Neurophysiology, 79, 27-35.

Breier, A. (1995). Serotonin, schizophrenia and antipsychotic drug action. Schizophrenia Research, 14, 187-202.

Brende, J., and Benedict, B. (1980). The Vietnam combat delayed stress response syndrome: Hypnotherapy of "dissociative symptoms." American Journal of Clinical Hypnosis, $23,34-40$.

Broad, C. D. (1925). The mind and its place in nature. London, England: Routledge and Kegan Paul. 
Brown, P. (1994). Toward a psychobiological model of dissociation and post-traumatic stress disorder. In Lynn, S., and Rhue, J. (Eds.). Dissociation: Clinical and theoretical perspectives. New York, NY: Guilford Press.

Bunge, M. (1980). The mind-body problem: A psychobiological approach. New York, NY: Pergamon Press.

Carlson, E. (1994). Studying the interaction between physical and psychological states with the Dissociative Experiences Scale. In Spiegel, D. (Ed.), Dissociation: Culture, mind, and body. Washington, DC: American Psychiatric Press.

Carlson, N. (1986). Physiology of behavior. Boston, MA: Allyn and Bacon.

Collier, B. (1972). Ketamine and the conscious mind. Anesthesia, 27, 120-134.

Collins, R. (1990). Cerebral cortex. In Pearlman, A., and Collins, R. (Eds.). Neurobiology of disease. New York, NY: Oxford University Press.

Cornman, J. (1981). A nonreductive identity thesis about mind and body. In Feinberg, J. (Ed.), Reason and responsibility. Belmont, CA: Wadsworth.

Dane, L. (1975-76). Astral travel: A psychological overview. Journal of Altered States of Consciousness, 2, 249-258.

Dennett, D. (1991). Consciousness explained. Boston, MA: Little, Brown.

Descartes, R. (1986). Meditations on first philosophy (J. Cottingham, Trans.). Cambridge, England: Cambridge University Press. (Original work published 1641)

Devinsky, O., Feldmann, E., Burrowes, K., and Bromfield, E. (1989). Autoscopic phenomena with seizures. Archives of Neurology, 46, 1080-1088.

Donvan, J. (Dir.). (1994). Life after death: Personal experiences. In Sawyer, D. (Producer), Turning point (television broadcast). New York, NY: American Broadcasting Company.

Einstein, A. (1961). Relativity. New York, NY: Crown.

Ellenson, G. (1985). Detecting a history of incest: A predictive syndrome. Social Casework, $66,525-532$.

Eyring, H., Walter, J., and Kimball, G. (1944). Quantum chemistry. New York, NY: Wiley.

Ferris, T. (1991, December 15). A cosmological event. New York Times Magazine, pp. 44 and $52-53$.

Floyd, K. (1996). ECT: TNT or TLC? A near-death experience triggered by electroconvulsive therapy. Journal of Near-Death Studies, 14, 187-195.

Folkman, S., Lazarus, R., Gruen, R., and DeLongis, A. (1986). Appraisal, coping, health status, and psychological symptoms. Journal of Personality and Social Psychology, 50, 571-579.

Gómez-Jeria, J. S., and Madrid-Aliste, C. (1996). Evolution and the relationship between brain and mind states. Journal of Near-Death Studies, 14, 251-272.

Gómez-Jeria, J. S., and Saavedra-Aguilar, J. C. (1994). A neurobiological model for neardeath experiences. II: The problem of recall of real events. Journal of Near-Death Studies, 13, 81-89.

Gordh, T., Karlsten, R., and Kristensen, J. (1995). Intervention with spinal NMDA, adenosine, and NO systems for pain modulation. Annals of Medicine, 27, 229-234.

Greer, J. (1994). "Return of the repressed" in the analysis of an adult incest survivor: A case study and some tentative generalizations. Psychoanalytic Psychology, 11, 545-561.

Grosso, M. (1976). Some varieties of out-of-body experience. Journal of the American Society for Psychical Research, 70, 179-193.

Grof, S., and Halifax, J. (1977). The human encounter with death. New York, NY: Dutton.

Halliday, D., and Resnick, R. (1970). Fundamentals of physics. New York, NY: Wiley.

Halpern, P. (1992). Cosmic wormholes: The search for interstellar shortcuts. New York, NY: Dutton.

Hawking, S. (1988). A brief history of time: From the big bang to black holes. New York, NY: Bantam.

Hergenhahn, B. (1986). An introduction to the history of psychology. Belmont, CA: Wadsworth.

Jackson, J. (1975). Classical electrodynamics. New York, NY: Wiley. 
Jansen, K. L. R. (1990). Neuroscience and the NDE: Roles for the NMDA-PCP receptor, the sigma receptor and the endopsychosins. Medical Hypotheses, 31, 25-29.

Jansen, K. L. R. (1997). The ketamine model of the near-death experience: A central role for the N-methyl-D-aspartate receptor. Journal of Near-Death Studies, 16, 5-26.

Khatami, M. (1978). Creativity and altered states of consciousness. Psychiatric Annals, $8,57-64$.

Kluft, R. (1990). Dissociation and subsequent vulnerability: A preliminary study. Dissociation, 3, 167-173.

Krishnan, V. (1999). The theory of essence [Letter]. Journal of Near-Death Studies, 18 , 65-66.

Landau, L., and Lifshitz, E. (1977). Quantum mechanics: Non-relativistic theory. Oxford, England: Pergamon Press.

Lahti, A., Koffel, B., LaPorte, D., and Tamminga, C. (1995). Subanesthetic doses of ketamine stimulate psychosis in schizophrenia. Neuropsychopharmacology, 13, 9-19.

Lazarus, R., and Folkman, S. (1984). Stress, appraisal, and coping. New York, NY: Springer.

Lester, M. (1989). The sum of the parts: Written dialogue in the treatment of incest. Journal of Independent Social Work, 4, 79-94.

Levine, I. (1978). Physical chemistry. New York, NY: McGraw-Hill.

Loewenstein, R., and Putnam, F. (1988). A comparison study of dissociative symptoms in patients with complex partial seizures, MPD, and posttraumatic stress disorder. Dissociation, 1, 17-23.

Lorrain, P., and Corson, D. (1970). Electromagnetic fields and waves. San Francisco, CA: Freeman.

Lothman, E., and Collins, R. (1990). Seizures and epilepsy. In Pearlman, A., and Collins, R. (Eds.), Neurobiology of disease. New York, NY: Oxford University Press.

Luria, A. (1973). The working brain: An introduction to neuropsychology. New York, NY: Basic Books.

Maltz, W. (1988). Identifying and treating the sexual repercussions of incest: A couples therapy approach. Journal of Sex and Marital Therapy, 14, 142-170.

Meduna, L. (1950). The effect of carbon dioxide upon the functions of the brain. In Carbon dioxide therapy. Springfield, IL: Charles C Thomas.

Misner, C., Thorne, K., and Wheeler, J. (1973). Gravitation. San Francisco, CA: Freeman.

Moody, R. (1975). Life after life. Covington, GA: Mockingbird Books.

Morse, M. (1983). A near-death experience in a 7-year-old child. American Journal of Diseases of Children, 137, 959-961.

Morse, M., Conner, D., and Tyler, D. (1985). Near-death experiences in a pediatric population. American Journal of Diseases of Children, 139, 595-600.

Morse, M., Castillo, P., Venecia, D., Milstein, J., and Tyler, D. (1986). Childhood near-death experiences. American Journal of Diseases of Children, 140, 1110-1114.

Morse, M., and Perry, P. (1990). Closer to the light: Learning from the near-death experiences of children. New York, NY: Villard.

Morse, M., Venecia, D., and Milstein, J. (1989). Near-death experiences: A neurophysiological explanatory model. Journal of Near-Death Studies, 8, 45-53.

Noyes, R. (1978). Near-death experiences: Their interpretation and significance. In Kastenbaum, R. (Ed.), Between life and death (pp. 73-88). New York, NY: Springer.

Noyes, R., and Kletti, R. (1976a). Depersonalization in the face of life-threatening danger: A description. Psychiatry, 39, 19-114.

Noyes, R., and Kletti. R. (1976b). Depersonalization in the face of life-threatening danger: An interpretation. Omega, 7, 103-114.

Pearlman, A., and Collins, R. (Eds.). (1990). Neurobiology of disease. New York, NY: Oxford University Press.

Penfield, W. (1955). The role of the temporal cortex in certain psychical phenomena. Journal of Mental Science, 101, 451-465. 
Penfield, W. (1975). The mystery of the mind. Princeton, NJ: Princeton University Press.

Penfield, W. and Rasmussen, T. (1950). The cerebral cortex of man: A clinical study of localization of function. New York, NY: Macmillan.

Persinger, M. (1995). Out-of-body-like experiences are more probable in people with elevated complex partial epileptic-like signs during periods of enhanced geomagnetic activity: A nonlinear effect. Perceptual and Motor Skills, 80, 563-569.

Persinger, M., and Makarec, K. (1993). Complex partial epileptic signs as a continuum from normals to epileptics: Normative data and clinical populations. Journal of Clinical Psychology, 49, 33-45.

Pierce, P. and Peroutka, S. (1990). Antagonist properties of d-LSD at 5-hydroxytryptamine-sub-2 receptors. Neuropsychopharmacology, 3, 503-508.

Popper, K. (1959). The logic of scientific discovery. London, England: Hutchinson.

Powers, W. (1990). Stroke. In Pearlman, A., and Collins, R. (Eds.). Neurobiology of disease. New York, NY: Oxford University Press.

Rice, P. (1987). Stress and health: Principles and practice for coping and wellness. Belmont, CA: Wadsworth.

Ring, K. (1980). Life at death: A scientific investigation of the near-death experience. New York, NY: Coward, McCann and Geoghegan.

Ring, K. (1984). Heading towards omega: In search of the meaning of the near-death experience. New York, NY: Morrow.

Rogo, D. S. (1984). Ketamine and the near-death experience. Anabiosis: The Journal of Near-Death Studies, 4, 87-96.

Ross, C. (1994). Dissociation and physical illness. In Spiegel, D. (Ed.), Dissociation: Culture, mind, and body. Washington, DC: American Psychiatric Press.

Saavedra-Aguilar, J., and Gómez-Jeria, J. (1989). A neurobiological model for near-death experiences. Journal of Near-Death Studies, 7, 205-222.

Sabom, M. B. (1982). Recollections of death: A medical investigation. New York, NY: Harper and Row.

Saletu, B. (1987). Brain function during hypnosis, acupuncture and transcendental meditation: Quantitative EEG studies. Advances in Biological Psychiatry, 16, 18-40.

Silon, B. (1992). Dissociation: A symptom of incest. Individual Psychology, 48, 155-164.

Steinberg, M. (1994). Systematizing dissociation: Symptomology and diagnostic assessment. In Spiegel, D. (Ed.), Dissociation: Culture, mind, and body. Washington, DC: American Psychiatric Press.

Stryer, L. (1978). Fluorescence energy transfer as a spectroscopic ruler. Annual Review of Biochemistry, 47, 819-846.

Stryer, L. (1988). Biochemistry. New York, NY: Freeman.

Sutherling, W., Crandall, P., Levesque, M., Darcey, T. and Barth, D. (1992). Physical interpretation of frontal lobe seizures: The dipole approximation and sensorimotor cortex. Advances in Neurology, 57, 339-347.

Taylor, R. (1992). Metaphysics. Englewood Cliffs, NJ: Prentice-Hall.

Thorne, K. (1994). Black holes and time warps: Einstein's outrageous legacy. New York, NY: Norton.

Viney, W. (1993) A history of psychology: Ideas and context. Boston, MA: Allyn and Bacon.

Wolf, F. A. (1988). Parallel universes: The search for other worlds. New York, NY: Simon and Schuster. 